\title{
The Effect of Administered Activity on Patient Radiation dose and Image Quality in SPECT at Korle-Bu Teaching Hospital
}

\author{
Issahaku Shirazu$^{1}$, Theophilus Sackey ${ }^{2}$, Ernest Kojo Eduful ${ }^{3}$ \\ ${ }^{1,2,3}$ Medical Radiation Physics Centre, Radiological \& Medical Sciences Research Institute, Ghana Atomic Energy \\ Commission, Ghana \\ ${ }^{1,2,3}$ National Centre for Radiotherapy and Nuclear Medicine, Department of Nuclear Medicine, Korle-Bu Teaching \\ Hospital, Accra Ghana
}

\begin{abstract}
The study discussed two parameters these include; administered activity (patients dose) and image quality. The aim is to determine the relationship between administered activity with resultant patient radiation dose and the quality of images produced. This will help make appropriate recommendation to the technologist and the nuclear medicine physician to produce images that would answer clinical question and at the same time maintain a balance with patient's radiation dose and its prognostic consequences. The study include both quadrant bar phantom study and patient image study in the form of static and dynamic studies. To determine image quality both the quadrant bar phantom and the patients images were assess by using SNR. The quadrant bar phantom was imaged by placing it on flood field uniform phantom which contained the radionuclide. The flood field uniformity phantom was filled with water and then an injected activity, which varied between 5 to $45 \mathrm{mCi}$ of Tc-99 m were added and mixed thoroughly by several shaking for about 5 minutes. Images of the quadrant bar phantom together with real patients' images with varied injected activities were analyzed. The study shows that as the administered activity increases from 5 to $45 \mathrm{mCi}$ the image quality increases significantly, which is based on the increase in SNR. Where the image quality increases from 17.06 to 22.29 in LEAP collimator and 13.56 to 21.51 in LEHR collimator using patients' static images. In addition, the image quality also increases from 18.26 to 20.44 in LEAP and 13.51 to 21.47 in LEHR collimator using quadrant bar phantom studies. Furthermore, in the of dynamic patients images, the SNR had minimal variation from 5.40 to 29.86 in LEAP and 7.11 to 21.99 in LEHR. Furthermore the reduction in administered activity increases the acquisition time from 7 to 20mins for LEAP collimator and 8 to 22 mins for LEHR collimator. In addition, with the phantom study, the acquisition time increases from 12 to 19 mins for LEAP and 13 to 15 mins for LEHR collimator. Dynamic studies varies slightly in acquisition time just approximately a min increase with increase in administered activity.
\end{abstract}

Keywords : Administered Activity, LEAP, LEHR, Quadrant Bar Phantom, SNR, Flood Field Uniformity Phantom

\section{INTRODUCTION}

SPECT imaging is the combination of computed tomography method and conventional nuclear medicine (NM) imaging technique. SPECT imaging procedure involve the use of radiopharmaceutical selection, injected dosage of radioactivity, collimator selection, imaging time and image quality [1]. The goal is to optimize each of the parameters to obtain an image quality that allows the nuclear medicine physician to answer the clinical question. The difficulty is that these parameters are not independent. Changing one parameter will affect the others [2]. There are a variety of radionuclides used in nuclear medicine. The radionuclide used in Ghana is $99 \mathrm{mTc}$ which is obtained from the $99 \mathrm{Mo} / 99 \mathrm{mTc}$ generator imported from South Africa and Turkey. It has a monoenergetic gamma emission at $140 \mathrm{keV}$ with a short half-life of 6 hours, and is easily tagged to chemical forms that allow imaging of many physiologic processes. Other radionuclides such as iodine-131 (131-I) and iodine-123 (123-I) are also used but with much less frequency. They have longer half-lives and higher energy gamma emissions [3].

In addition, because $99 \mathrm{mTc}$ is used so frequently the world over, most nuclear medicine cameras are designed 
for imaging using radionuclide. Thus, the thickness of the $\mathrm{NaI}(\mathrm{Tl})$ is commonly $9.5 \mathrm{~mm}$. This results in an intrinsic efficiency of about $92 \%$. As the energy of the photons increase, the linear attenuation coefficient decreases and so does the detection efficiency. The selection of radionuclides that have energy close to 140 $\mathrm{keV}$ will result in better quality images for less radiation dose. An example would be the use of 123I (160 keV) rather than 131I (364 keV) for the post-surgical evaluation of patients with thyroid cancer [4].

Finally, some $99 \mathrm{mTc}$ radiopharmaceuticals used for the same purpose have different uptake and clearance characteristics that would make one better than the other in terms of radiation dose. For instance, the renal imaging agent 99mTc-MAG3 has higher uptake and more rapid clearance than $99 \mathrm{mTc}$-DTPA which is also used for renal imaging. For the same injected dosage, the 99mTc-MAG3 would be the preferred radiopharmaceutical [5]. Changing one parameter in a nuclear medicine procedure will affect all others [6]. It is critical to understand all of the changes that occur when modifying a single parameter. Encouraging patients to drink liquids and void frequently will also help to reduce the radiation dose for radiopharmaceuticals cleared by the kidneys [7].

\section{AIM}

The main aim was to determine the relationship between administered activity, patient radiation dose and image quality and to make appropriate recommendation to the technologist and the nuclear medicine physician to answer clinical question and at the same time reduces patient's radiation dose and its prognostic consequences.

\section{LITERATURE REVIEW}

A method of measuring the image quality of medical imaging equipment is considered within the framework of statistical decision theory [8]. In this approach, images are regarded as random vectors and image quality is defined in the context of the image information available for performing a specified detection or discrimination task [8]. These approach provides a means of measuring image quality, as related to the detection of an image detail of interest, without reference to the actual physical mechanisms involved in image formation and without separate measurements of signal transfer characteristics or image noise [9].
The measurement does not, however, consider deterministic errors in the image; there is the need for a separate evaluation of imaging modalities where there are of concern. The detectability of an image detail can be expressed in terms of the ideal observer's signal-tonoise ratio at the decision level. Often a good approximation to this SNR can be obtained by employing sub-optimal observers, whose performance correlates well with the performance of human observers as well. Therefore, the utilization of sub-optimal observers is advised. Hence, image quality are assessed using the relation between process signals to the noise known as SNR [8].

Signal to Noise Ratio in terms of pixel value is define as the reciprocal of the coefficient of variation $(\mathrm{CV})$, i.e., the ratio of mean to standard deviation of a signal or measurement. It is also described in voxel format as a measured of the ratio of the mean to the standard deviation of voxel values express mathematically as:

$$
S N R=\frac{\mu}{\sigma}
$$

where $\mu$ is the signal mean or expected value and $\sigma$ is the standard deviation of the noise, or an estimate thereof [10]. This is a useful for photon counts in image processing, where the SNR of an image is usually calculated as the ratio of the mean pixel value to the standard deviation of the pixel values over a given neighborhood or background. It can also be defined as the square of the signal mean value to the standard deviation.

$$
S N R=\sqrt{\frac{\mu^{2}}{\sigma^{2}}}=\sqrt{\frac{r}{1-r}}
$$

where $r$ is the correlation coefficient.

SNR are used to assess the quality of medical images in all form of common general nuclear medicine imaging examinations. These include:
$\checkmark$ Bone Scintigraphy
$\checkmark$ Parathyroid Scintigraphy
$\checkmark$ Thyroid Scintigraphy and Uptake
$\checkmark$ Thyroid therapy
$\checkmark$ Lung (V/Q) Scintigraphy
$\checkmark$ Hepatobiliary Scintigraphy
$\checkmark$ Renal Scintigraphy 


\section{METHODS AND MATERIAL}

The materials used included:
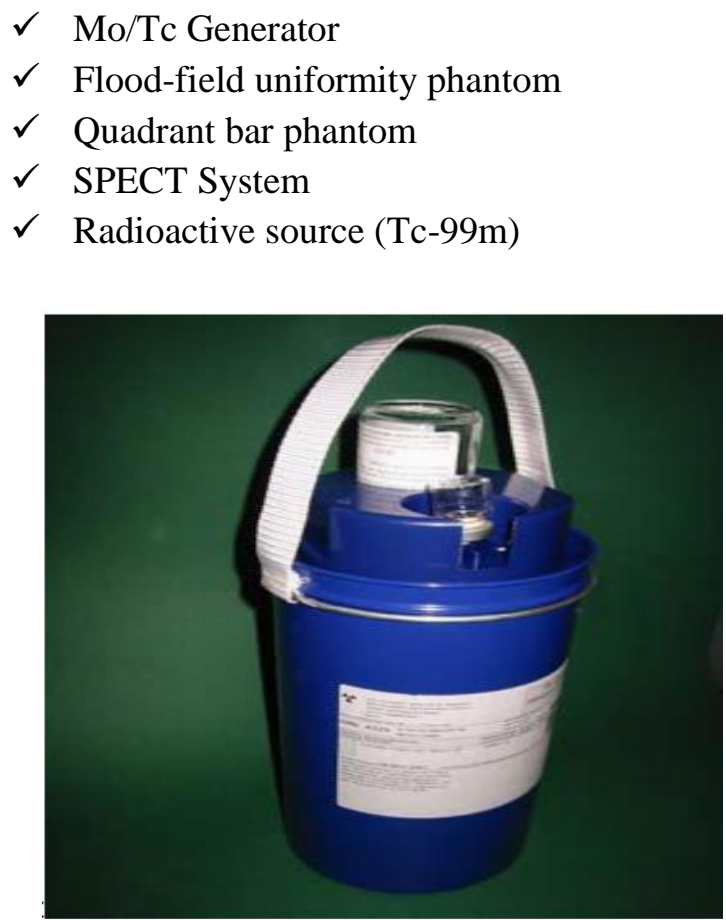

Figure 1. Mo/Tc Generator

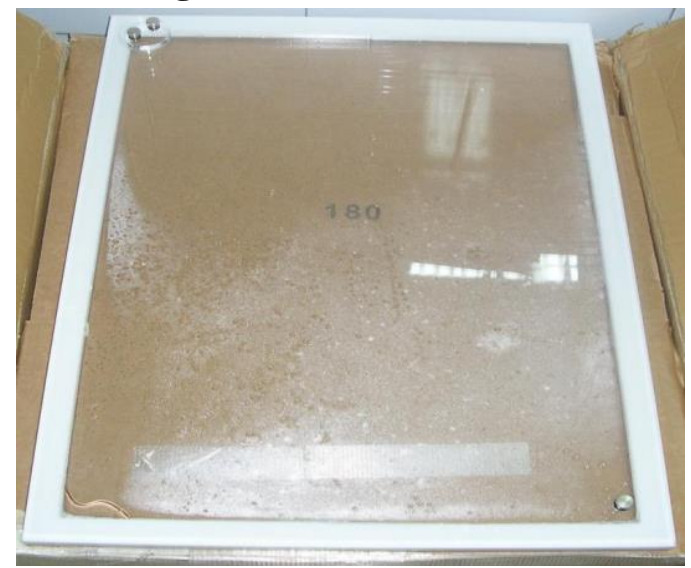

Figure 2. Flood-field uniformity phantom

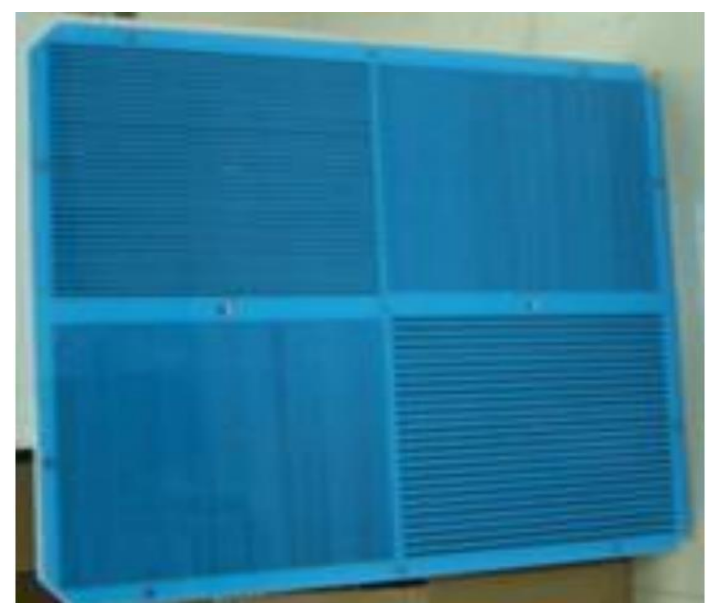

Figure 3. Quadrant bar phantom

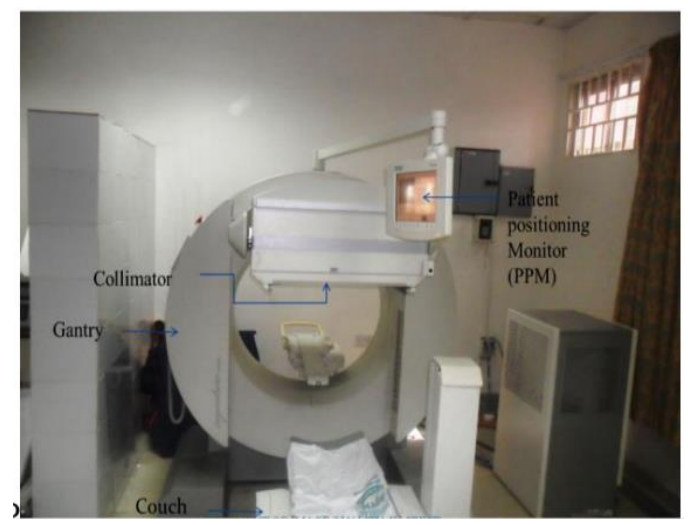

Figure 4. SPECT System

\section{A. Method}

The study covered two parameters which include: administered activity (patient dose) and image quality. Signal to noise ratio (SNR) was used to assess image quality both on patients' static and dynamic images and quadrant bar phantom (QBP) [11]. Before imaging the flood field uniformity phantom was half filled with water and then an injected activity were varied between 5 to $45 \mathrm{mCi}$ of $\mathrm{Tc}-99 \mathrm{~m}$ were added. Each time the radio-active material was mixed thoroughly by several shaking for about 5 minutes and the phantom carefully filled with water to avoid bubbles in it. It was turned up by inversion and then topped up to leave a small residual air bubble at the air space provided. Then, the QBP was placed on the flood field uniform phantom and place under the camera for imaging as a unit refer to as quadrant bar uniformity phantom. In addition patients' static and dynamic images were obtain from the archiving system and its SNR access. This was done by measuring the average count and variance per fix matrix and object-collimator distance. In addition the background radiation were also estimated. Hence by using equation 3 SNR was determined.

\section{B. Procedure}

The injected or administered activity of Tc-99 m were varied five time, that is less than 10, 11-20, 21-30, 3140, more than $41 \mathrm{mCi}$ while, keeping all parameters constant. These were repeated five times and the acquire images labeled base on the injected activities. The quadrant bar uniformity phantom (quadrant bar phantom on flood field uniformity phantom) was positioned on the SPECT gamma camera patient bed, by ensuring that the flat axis of the phantom was positioned parallel to 
the axis of the gamma camera detector, within the field of view (FOV) of the detector. Quadrant Bar phantom on the flood field phantom together were placed under the detector. The images were acquire using a constant matrix size, count density and object image distance.

In addition, from the acquired patients existing images with varied injected Tc-99m obtained from the archiving system were also grouped in line with that of the injected activities in the flood field uniformity phantom that is less than 10, 11-20, 21-30, 31-40, more than 41 mCi. Signal to noise ratio of these images were estimated and analyzed to determine the variations between the quality of the images produce and the dose received by the patients during the image studies.

\section{Image Quality}

Image quality was assess by using the homogeneous volume method to estimate signal to noise ratio. This was done by finding a homogeneous area within the image and compute the process signal (average) and the noise (standard deviation) $[5,13]$. The ratio of the mean to the standard deviation gives an estimate for the signal to noise ratio of the image. The signal to noise ratio in this case is the reciprocal of the coefficient of variation $(\mathrm{CV})$, a statistical measure which is often used in statistics to describe the amount of variability relative to the mean [14]. Hence CV parameters was also estimated and because coefficient of variation is unitless, it was used, instead of the standard deviation to compare the spread of data sets, since the data has varied means. In this case the CV was determine as the reciprocal of SNR and a measure of the magnitude of a data set relative to the standard deviation and was also describe as a relative standard deviation (RSD) [13]. The result of this will be described as larger, if SNR has a magnitude of the signal or process average been large relative to the noise as measured standard deviation. In addition, if SNR is large, then the signal is deemed to be significant and not just random variation. Hence, large value of signal to noise shows better image quality and vice versa.

SNR was estimated using the mathematically expression:

$$
\text { SNR }=\frac{\mu(\text { Signal })-\mu(\text { background })}{\text { Noise }(\sigma)}
$$

\section{RESULTS AND DISCUSSION}

\section{A. Pictorial Representation}

Images of quadrant bar phantom on flood field uniformity phantom are shown below.

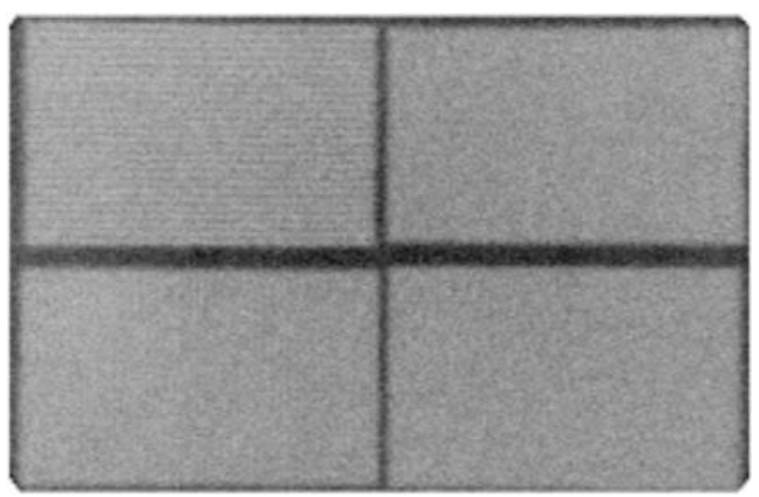

Figure 5. Activity of less than $10 \mathrm{mCi}$

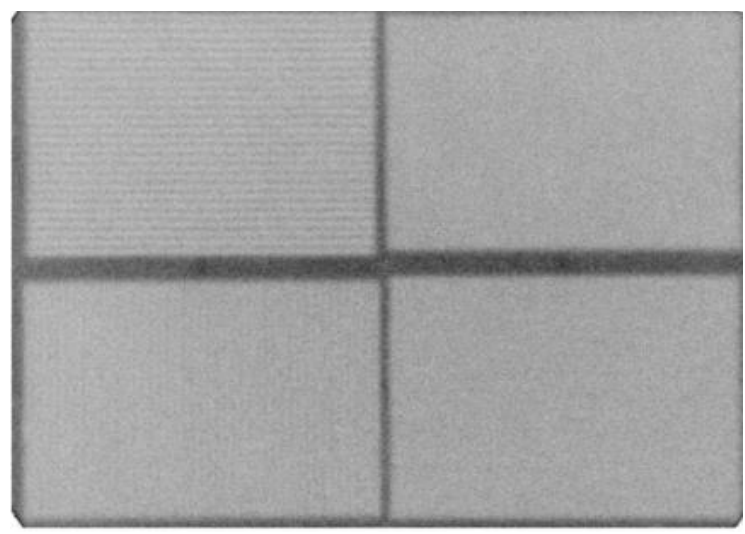

Figure 6. Activity of $11-20 \mathrm{mCi}$

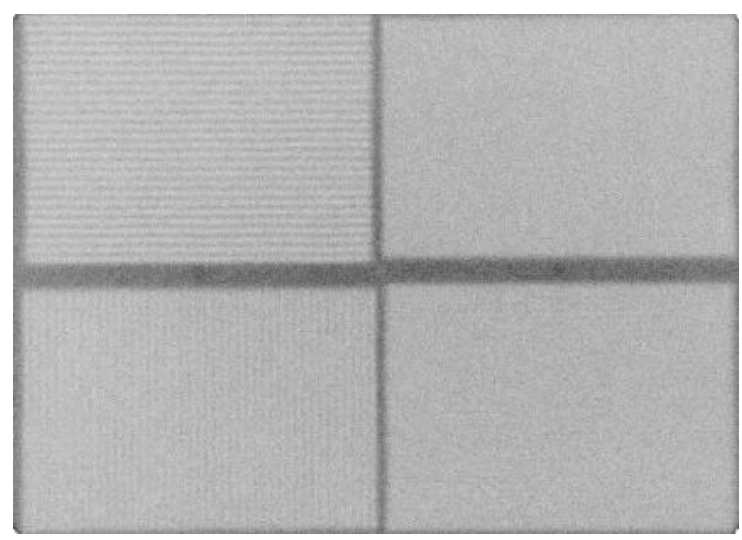

Figure 7. Activity of $21-30 \mathrm{mCi}$ 


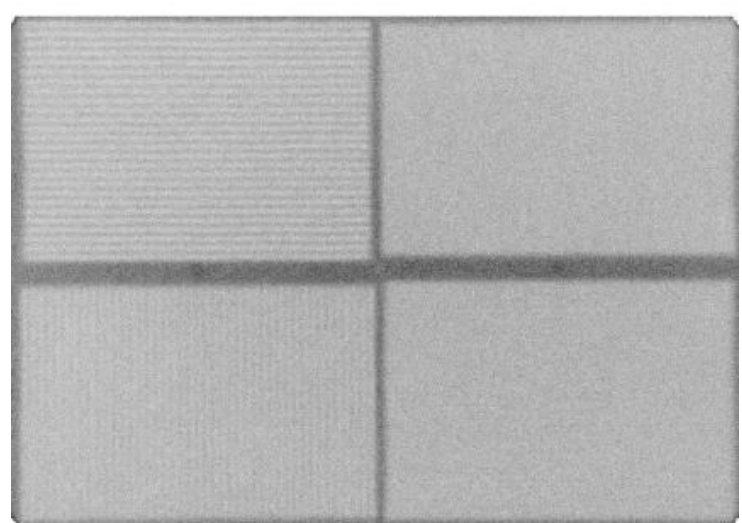

Figure 8. Activity of $31-40 \mathrm{mCi}$

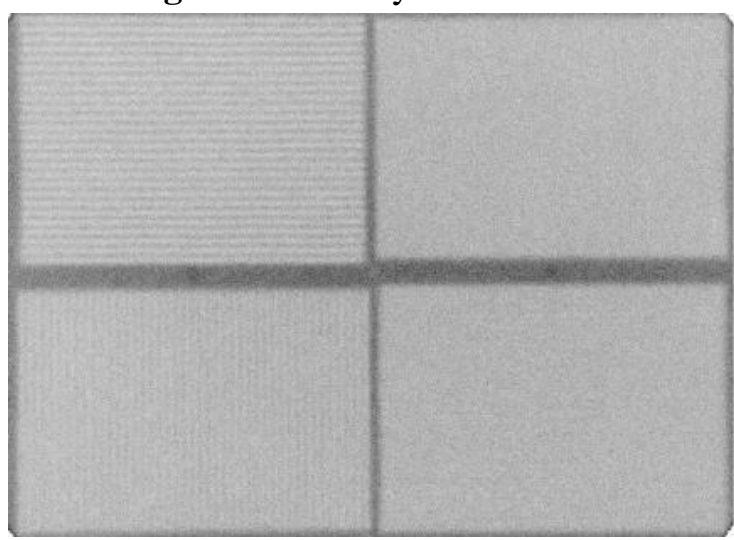

Figure 9. Activity of more than $41 \mathrm{mCi}$
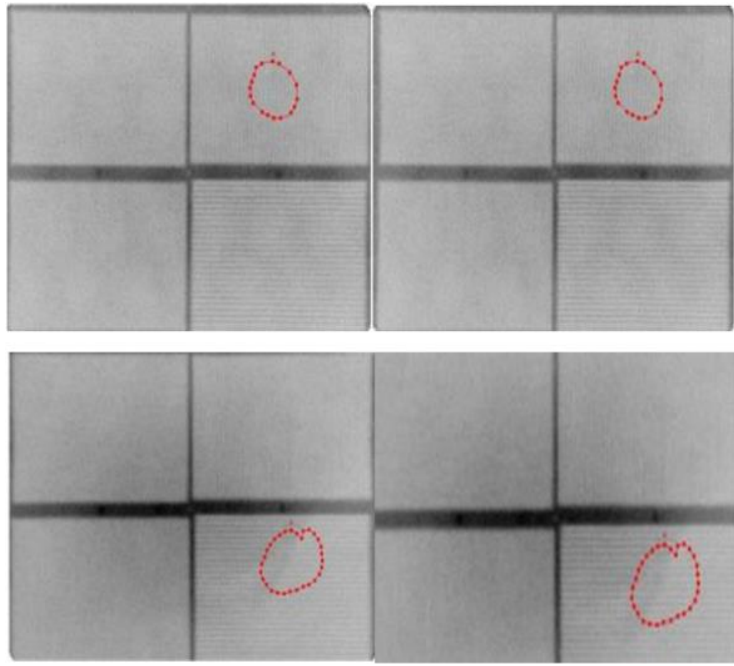

Figure 10. Measurements of SNR

\section{Table of Values}

The results of measured parameters are tabulated below

Table 1. Measurements of SNR on static Patients images

\begin{tabular}{|c|c|c|c|c|c|}
\hline & $\begin{array}{c}\text { Varied Dose } \\
\text { Parameters } \\
\mathrm{mCi} \\
\end{array}$ & $\begin{array}{l}\text { Average Counts per } \\
\text { Matrix Size (cts/pixel) }\end{array}$ & Variance & SNR & $\begin{array}{c}\text { Acquisition } \\
\text { Time } \\
\text { (mins) }\end{array}$ \\
\hline \multirow{5}{*}{ 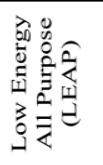 } & $<10$ & 392.14 & 528.29 & 17.05698 & 19.65 \\
\hline & $11-20$ & 524.94 & 791.53 & 18.66122 & 16.37 \\
\hline & $21-30$ & 657.39 & 1091.41 & 19.89679 & 13.27 \\
\hline & $31-40$ & 781.07 & 1319.06 & 21.50523 & 10.12 \\
\hline & $>40$ & 867.54 & 1675.11 & 22.28461 & 7.27 \\
\hline \multirow{5}{*}{ 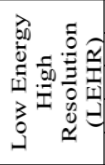 } & $<10$ & 403.81 & 886.96 & 13.55977 & 22.43 \\
\hline & $11-20$ & 520.62 & 906.28 & 17.29060 & 19.03 \\
\hline & $21-30$ & 653.75 & 1124.86 & 19.49165 & 15.16 \\
\hline & $31-40$ & 774.76 & 1632.61 & 20.17079 & 11.51 \\
\hline & $>40$ & 867.43 & 1876.64 & 21.51364 & 8.42 \\
\hline
\end{tabular}

Table 2. Measurements of SNR on quadrant bar phantom

\begin{tabular}{|c|c|c|c|c|c|}
\hline & $\begin{array}{c}\text { Varied Dose } \\
\text { Parameters } \\
\text { mCi }\end{array}$ & $\begin{array}{l}\text { Average Counts per } \\
\text { Matrix Size (cts/pixel) }\end{array}$ & Variance & SNR & $\begin{array}{c}\text { Acquisition } \\
\text { Time } \\
\text { (mins) }\end{array}$ \\
\hline \multirow{5}{*}{ 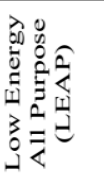 } & $<10$ & 434.21 & 565.67 & 18.25946 & 18.57 \\
\hline & $11-20$ & 471.45 & 598.95 & 19.26645 & 16.11 \\
\hline & $21-30$ & 495.94 & 642.11 & 19.57143 & 15.45 \\
\hline & $31-40$ & 518.81 & 682.52 & 19.85496 & 13.17 \\
\hline & $>40$ & 526.24 & 714.76 & 20.44444 & 12.36 \\
\hline \multirow{5}{*}{ 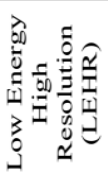 } & $<10$ & 512.79 & 1440.78 & 13.50869 & 14.57 \\
\hline & $11-20$ & 506.09 & 1271.83 & 14.19209 & 14.39 \\
\hline & $21-30$ & 542.59 & 1142.49 & 16.05296 & 14.27 \\
\hline & $31-40$ & 544.04 & 942.54 & 17.72117 & 14.20 \\
\hline & $>40$ & 602.34 & 786.6 & 21.47380 & 13.29 \\
\hline
\end{tabular}

Table 3. Measurements of SNR for dynamic study

\begin{tabular}{|c|c|c|c|c|c|}
\hline & $\begin{array}{l}\text { Varied Dose } \\
\text { Parameters }\end{array}$ & $\begin{array}{l}\text { Average Counts per } \\
\text { Matrix Size (ctspivel) }\end{array}$ & Variance & SNR & $\begin{array}{c}\text { Acquisition Time } \\
\text { (mins) }\end{array}$ \\
\hline \multirow{5}{*}{ 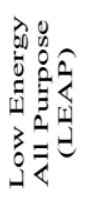 } & $<10$ & 7788.94 & 68047.9 & 5.403035 & 11.29 \\
\hline & $11-20$ & 32.04 & 35.21 & 9.811482 & 11.09 \\
\hline & $21-30$ & 126.47 & 166.15 & 11.98118 & 10.47 \\
\hline & $31-40$ & 477.57 & 1588.89 & 18.74904 & 10.29 \\
\hline & $>41$ & 1955.15 & 10874.3 & 29.8587 & 10.09 \\
\hline \multirow{5}{*}{ 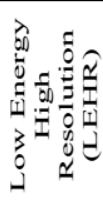 } & $<10$ & 51.2 & 51.83 & 7.111111 & 11.27 \\
\hline & $11-20$ & 199.75 & 288.16 & 11.76384 & 11.04 \\
\hline & $21-30$ & 854.66 & 1983.9 & 19.18859 & 10.20 \\
\hline & $31-40$ & 3038.24 & 31994.8 & 21.98574 & 10.10 \\
\hline & $>41$ & 7638.65 & 87046.5 & 25.89022 & 9.50 \\
\hline
\end{tabular}

\section{B. Graphical Representation}

\section{VARIATION OF INJECTED ACTIVITY}

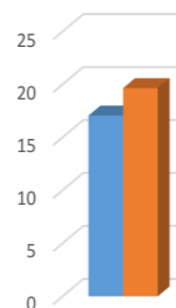

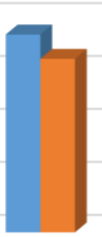

1020

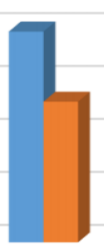

21-30

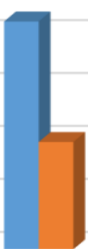

$31-40$

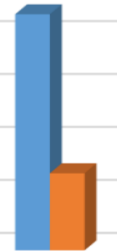

$>40$
mSNR $\quad$ Acquisition Time (mins)

Figure 11. SNR and A. time due to increase dose for LEAP on static images 


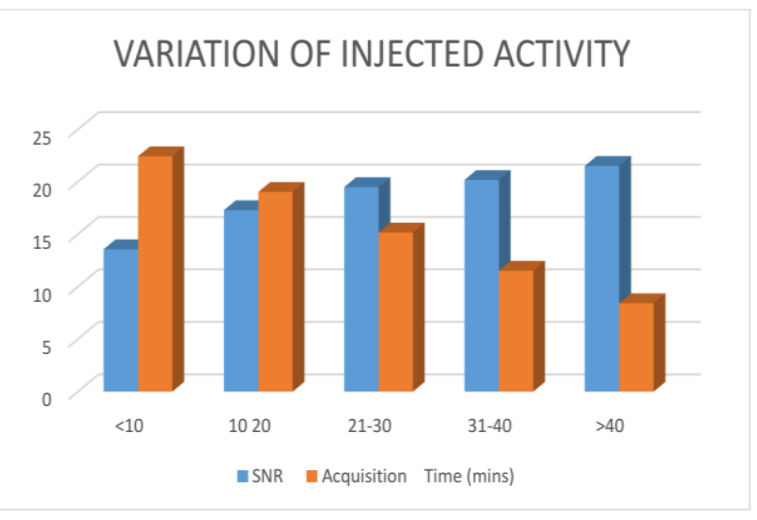

Figure 12. SNR and A. time due to increase dose for LEHR on static images

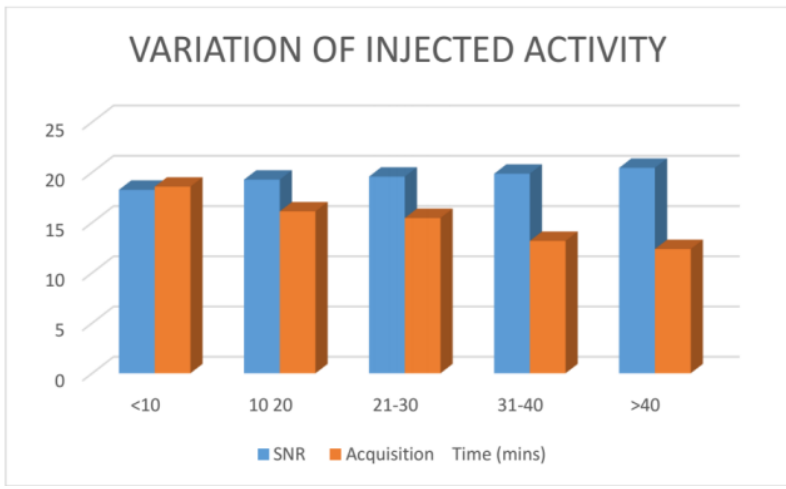

Figure 13. SNR and A. time due to increase dose for LEAP on QBP images

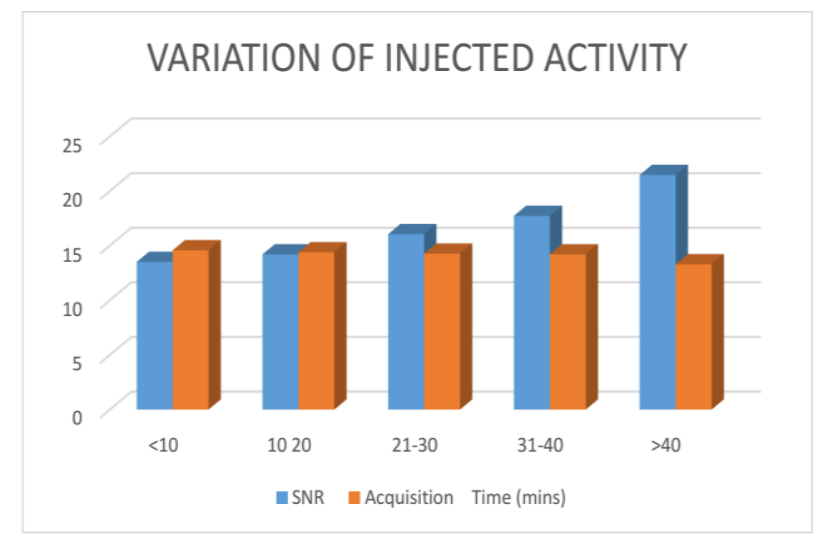

Figure 14. SNR and A. time due to increase dose for LEHR on QBP images
VARIATION OF INJECTED ACTIVITY

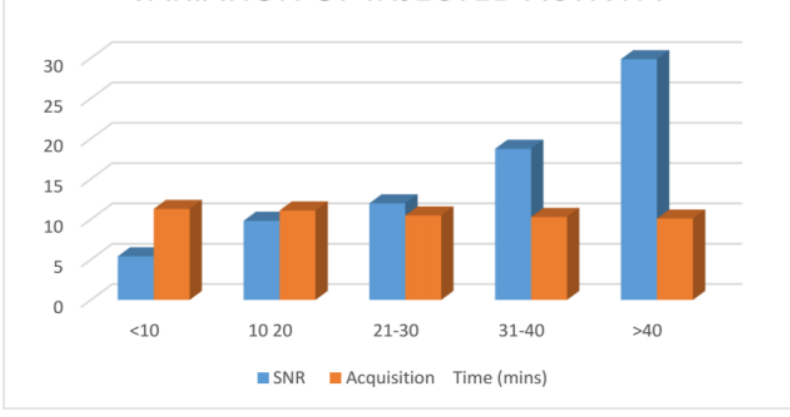

Figure 15. SNR and A. time due to increase dose for LEAP on dynamic images

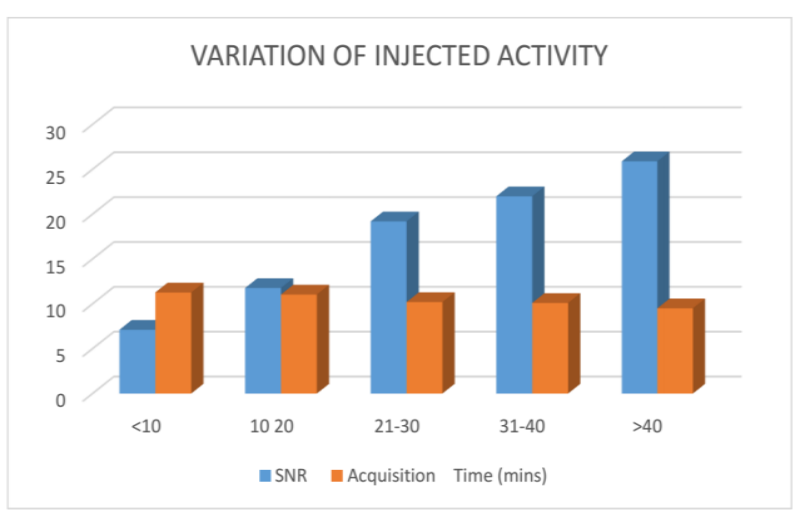

Figure 16. SNR and A. time due to increase dose for LEHR on dynamic images

\section{Discussion}

The study include patient radiation exposure as a results of administered dose and image quality. The administered dose varied from $5 \mathrm{mCi}$ to $45 \mathrm{mCi}$. Three different imaging procedures were used to analyze images quality based on SNR, this include, dynamic imaging procedure, static imaging procedure and quadrant bar phantom images. The study shows that as the administered activity increases from 5 to $45 \mathrm{mCi}$ the image quality increases significantly based on the significant increase in SNR. The SNR increases from 17.06 to 22.29 in LEAP collimator and 13.56 to 21.51 in LEHR collimator using patients' static images. In addition, the signal to noise ratio increases from 18.26 to 20.44 in LEAP and 13.51 to 21.47 in LEHR collimator using quadrant bar phantom studies. Furthermore, in the case of the dynamic patients' images, the SNR increases from 5.40 to 29.86 in LEAP and 7.11 to 21.99 in LEHR. Furthermore the reduction in administered activity increases the acquisition time from 7 to $20 \mathrm{mins}$ in LEAP collimator and 8 to 22 mins for LEHR collimator. In addition, with the phantom study, the acquisition time 
increases from 12 to 19 mins in LEAP and 13 to 15 mins in LEHR collimator. Dynamic studies varies slightly in acquisition time just approximately a min increase with increase in administered activity. Details of these variations are shown in table 1 to table three. Fig 11 to Fig. 16 shows the relationship between acquisition time and image quality in term of SNR as a function of the administered or injected activities.

\section{Analysis}

The relationship between patient radiation exposure in relation to administered activity and image quality is clearly measured as a function of signal to noise ratio and the radionuclide used. The results from this study shows that there is a positive correlation between the injected activity and the quality of images produced [8, 15]. This would help advice the technologists Nuclear Medicine physicians in their daily operations. Especially in reference to how much dose should be given in other not to unnecessary exposure the patient and at the same time that would not affect the quality of images produced. In addition, the amount of radioactivity injected into the patient is directly proportional to the radiation dose delivered [16]. The greater the activity injected, the higher the radiation dose to the patient and the smaller the noise, and hence the higher the signal to noise ratio This was more pronounce in static imaging procedures but minimal in dynamic study as shown in Fig 15 and Fig 16. However, in dynamic studies results of reducing the injected radioactivity is not as simple as static studies. Most dynamic imaging procedures are severely compromised by a reduction in injected radioactivity. Dynamic imaging procedures that have a short time per frame need to have enough counts in each image to visualize the process of interest and not compromise the quantitative results by the analysis of overly noisy data $[2,16]$. When assigning a quantity of radioactivity to be injected, the clinical question at hand must be considered. The smallest amount of radioactivity that will provide images that will answer that question should be used [16].

The goal is to allow for faster imaging and increased throughput. In recent times software programs are used in addition to other measure taken measures taken before and during imaging. However, these software are done to reduce the injected dosage while keeping the imaging time at what was used without the image restoration software [13, 17]. In some instances these software packages may provide for as much as a $50 \%$ reduction in radiation dose to the patient.

Each procedure in nuclear medicine should be reviewed with the goal of reducing patient dose. The lowest injected dosage should be used that will answer the clinical question. The physical characteristics of the imaging system such as detector efficiency, collimator resolution and sensitivity, and software for resolution recovery should be considered when defining nuclear medicine procedures with the goal of reducing radiation dose to the patient [17]

Reducing the administered activity of a radiopharmaceutical will reduce patient radiation exposure, but it will also affect the examination's imaging characteristics and may lower image quality. In the simplest terms, reducing administered activity will lower the count rate $[15,13,18]$. If during the examination's other acquisition parameters are unchanged, this may result in reduced visibility of the organ(s) of interest, increased image noise and limited detection of disease. If imaging times are increased to compensate for lower count rates, then the examination may become more susceptible to patient motion artifacts. The impact of lower administered activities may be greater in certain classes of patients, such as those with large body habitus, in whom count rates are further reduced by soft-tissue attenuation. It must be noted that universally accepted guidelines that optimize imaging by accounting for body habitus, (e.g., employing weight-based administered activities or adjusting gamma camera acquisition parameters according to an individual patient's body type) do not exist; thus, care must be taken in order to maintain sufficient diagnostic image quality when using lower administered activities. The adverse impact of reduced administered activity on image quality can be mitigated by using new camera technologies and software reconstruction algorithms and techniques [17, 19].

\section{REFERENCES}

[1]. Donohoe KJ, Brown ML, Collier BD, et al. Procedure Guidelines for Skeletal Scintigraphy, version 3.0.

[2]. Guidelines and Standards Committee of the ACR Commission on Nuclear Medicine in collaboration 
with the SPR. ACR-SPR Practice Guideline for the Performance of Adult and Pediatric Skeletal Scintigraphy (Bone Scan). American College of Radiology and Society of Pediatric Radiology, 2012.

[3]. Roberts CC, Weissman BN, Appel M, et al. Expert Panel on Musculoskeletal Imaging. ACR Appropriateness Criteria ${ }^{\circledR}$ Metastatic Bone Disease (revised). American College of Radiology, 2012.

[4]. Expert Panel on Musculoskeletal Imaging. ACR Appropriateness Criteria ${ }^{\circledR} \quad$ Musculoskeletal Imaging Criteria. American College of Radiology (ACR), 2008.

[5]. Guidelines and Standards Committee of the ACR Commission on Nuclear Medicine in Collaboration with the SPR. ACR -SPR Practice Guideline for the Performance of Parathyroid Scintigraphy. American College of Radiology and Society of Pediatric Radiology, 2009.

[6]. Greenspan BS, Dillehay G, Intenzo C, et al. SNM Practice Guideline for Parathyroid Scintigraphy 4.0. J Nuc Med Tech, 2012; 40:2:1.

[7]. Hinde E, Ugar O, Fuster D, et al. 2009 EANM Parathyroid Guidelines. Eur J Nucl Med Mol Imaging, 2009; 36:1201-1216.

[8]. Guidelines and Standards Committee of the Commission on Nuclear Medicine in collaboration with the SPR and SNM. ACR-SNM-SPR Practice Guideline for the Performance of Thyroid and Scintigraphy and Uptake Measurements. American College of Radiology, Society of Nuclear Medicine and Molecular Imaging, Society of Pediatric Radiology, 2009.

[9]. Balon HR, Silberstein EB, Meier DA, et al. Society of Nuclear Medicine Procedure Guideline for Thyroid Scintigraphy V3.0. Society of Nuclear Medicine and Molecular Imaging, 2006.

[10]. Gharib H, Papini E, Paschke R, et al. American Association of Clinical Endocrinologists, Associazione Medici Endocrinology, and European Thyroid Association Medical Guidelines for Clinical Practice for the Diagnosis and Management of Thyroid Nodules, 2010; 16:468475.

[11]. Cooper DS, Doherty GM, Haugen BR, et al. Revised American Thyroid Association Management Guidelines for Patients with Thyroid
Nodules and Differentiated Thyroid Cancer. Thyroid, 2009; 19:1167-1214.

[12]. Silberstein EB, Alavi A, Balon HR, et al. Society of Nuclear Medicine Procedure Guidelines for Scintigraphy for Differentiated Papillary and Follicular Thyroid Cancer. Society of Nuclear Medicine and Molecular Imaging, 2006.

[13].Parker JA, Coleman RE, Grady E, et al. SNM Practice Guideline for Lung Scintigraphy 4.0. J Nucl Med Tech, 2012, 40:1:57-6

[14]. Guidelines and Standards Committee of the ACR Commission on Nuclear Medicine in collaboration with the SPR. ACR-SPR Practice Guideline for the Performance of Pulmonary Scintigraphy. American College of Radiology and Society of Pediatric Radiology, 2009.

[15]. Bettmann MA, Baginski SG, White RD, et al. Expert Panel on Cardiac Imaging. ACR Appropriateness Criteria ${ }^{\circledR}$ Acute Chest Pain Suspected Pulmonary Embolism. American College of Radiology, 2011.

[16].Leung AN, Bull TM, Jaeschke R, et al. An Official American Thoracic Society/Society of Thoracic Radiology Clinical Practice Guideline: Evaluation of Suspected Pulmonary Embolism In Pregnancy. Am J Respir Crit Care Med, 2011, 184:1200-1208.

[17].Bajc M, Neilly JB, Miniati M, et al. EANM guidelines for ventilation/perfusion Scintigraphy Part 1. Pulmonary Imaging with Ventilation/Perfusion Single Photon Emission Tomography. Eur J Nucl Med Mol Imaging, 2009, 36:1356-1370.

[18]. Bajc M, Neilly JB, Miniati M, et al. EANM Guidelines for Ventilation/Perfusion Scintigraphy Part 2. Algorithms and Clinical Considerations for Diagnosis of Pulmonary Emboli with V/PSPECT and MDCT. Eur J Nucl Med Mol Imaging, 2009, 36:1528-1538.

[19]. Guidelines and Standards Committee of the ACR Commission on Nuclear Medicine in collaboration with the SPR and the SNM. ACR-SNM-SPR Practice Guideline for the Performance of Scintigraphy for Inflammation and Infection. American College of Radiology of Pediatric Radiology, 2009. ACR-SNM-SPR Practice Guideline for the Performance of Scintigraphy for Inflammation and Infection 